\title{
Placental-Site Gestational Trophoblastic Tumor
}

National Cancer Institute

\section{Source}

National Cancer Institute. Placental-Site Gestational Trophoblastic Tumor. NCI

Thesaurus. Code C3757.

A rare gestational trophoblastic tumor characterized by the presence of a diffuse cellular infiltrate composed of intermediate trophoblasts and cytotrophoblasts and by the absense of a significant population of syncytiotrophoblasts. 